\title{
GESTIÓN ADMINISTRATIVA DE APLICACIONES MÓVILES Y SU EFECTO EN LA COMERCIALIZACIÓN DE PRODUCTOS DE CONSUMO MASIVO EN EL CANTÓN QUEVEDO
}

\section{ADMINISTRATIVE MANAGEMENT OF MOBILE APPLICATIONS AND ITS EFFECT ON THE MARKETING OF MASS CONSUMER PRODUCTS IN THE QUEVEDO CANTON}

\author{
José Sólis Chang, Ing. \\ Ingeniero en Sistemas Administrativos Computarizados (Ecuador). \\ Alumni de la Universidad Técnica Estatal de Quevedo, Quevedo, Ecuador. \\ jmsch90@hotmail.com \\ Xavier Bucheli Espinoza, Mgtr. \\ https://orcid.org/0000-0001-5210-2322 \\ Magister en Mercadotécnica (Ecuador). \\ Docente de la Universidad Técnica Estatal de Quevedo, Quevedo, Ecuador. \\ nbucheli@uteq.edu.ec \\ Nelly Manjarrez Fuentes, Ph.D. \\ https://orcid.org/0000-0002-7615-3906 \\ Doctora en Ciencias Económicas (Cuba). \\ Docente de la Universidad Técnica Estatal de Quevedo, Quevedo, Ecuador. \\ nmanjarrez@uteq.edu.ec
}

\section{ARTÍCULO DE INVESTIGACIÓN}

Recibido: 19 de marzo de 2021

Aceptado: 23 de marzo de 2021

\section{RESUMEN}

La actual gestión administrativa de las empresas que usan aplicaciones móviles en Quevedo, se viene realizando sin un modelo de gestión definido. Al analizar la situación de estas empresas se define varios problemas, como: la ausencia de herramientas administrativas, la inadecuada administración de los negocios, evidenciando la falta de una estructura organizacional y conocimiento tecnológicos. La planificación y direccionamiento del modelo de negocios fue analizado a través de varios procesos y acciones que incentiven la adopción de una aplicación móvil para la comercialización de productos de consumo masivo. El objetivo de la investigación es evaluar la incidencia de la gestión administrativa de aplicaciones móviles y su efecto en la compra y entrega de productos de consumo masivo. El análisis de la gestión administrativa y la 
implementación tecnológica permitirá visualizar con mayor claridad los objetivos, debido a que los consumidores actuales no se le debe ofrecer productos o servicios sino conocimiento y experiencia, el presente trabajo plantea mejorar la gestión de los procesos administrativos y el desarrollo de una aplicación móvil para brindar el servicio de compra y entrega a domicilio de productos de consumo masivo. En este sentido, se encamina una propuesta de organización de procesos administrativos y operativos con el fin de incrementar la eficiencia operacional.

Palabras claves: gestión administrativa, aplicación móvil, negocio en línea

\section{ABSTRACT}

The current administrative management of companies that use mobile applications in Quevedo has been carried out without a defined management model. When analyzing the situation of these companies, several problems are defined, such as: the absence of administrative tools, inadequate business administration, evidencing the lack of an organizational structure and technological knowledge. The planning and directing of the business model was analyzed through various processes and actions that encourage the adoption of a mobile application for the commercialization of mass consumer products. The objective of the research is to evaluate the incidence of administrative management of mobile applications and its effect on the purchase and delivery of mass consumer products. The analysis of administrative management and technological implementation will allow the objectives to be seen more clearly, because current consumers should not be offered products or services but knowledge and experience, this work proposes to improve the management of administrative processes and the development of a mobile application to provide the service of purchase and home delivery of mass consumer products. In this sense, a proposal is directed to organize administrative and operational processes in order to increase operational efficiency.

Keywords: administrative management, mobile application, online business

\section{INTRODUCCIÓN}

La gestión administrativa implica un nivel elevado de exigencia tanto en el desarrollo de funciones como planificación, organización, coordinación, dirección y control de la organización, como para una mejor gerencia de los bienes de la empresa, entre ellos material, financiero y humano, orientado a la consecución de metas y objetivos trazados por el área directriz de la empresa. Actualmente estamos viviendo en la era tecnológica donde los principales beneficiados de estos avances son las personas y empresas, un ejemplo de estos avances son los dispositivos móviles como los smartphone y tablet son el principal ejemplo de tendencia tecnológica. Hoy en 
día las empresas, pymes y negocios locales se adaptan al avance tecnológico, según (Villacis, 2011) se ven obligadas a disponer de herramientas que le ayuden a expandir su funcionamiento.

Es aquí donde nace el "Paradigma de la Ubicuidad" que es el concepto de "estoy aquí y necesito procesarlo ya", introduciendo con esto, el concepto de movilidad. Con lo anterior, se puede afirmar que existen empresas, procesos y personas que requieren consultar, registrar y manipular la información de sus negocios en tiempo real sin importar el lugar en donde se en La principal herramienta de los dispositivos móviles son las aplicaciones móviles por aquello en los últimos años se ha observado su proliferación, estas cumplen funciones dentro de nuestro teléfono, ya sea para comunicarnos, entretenernos o ayudarnos, asimismo permiten a empresas ofrecer nuevos servicios a sus clientes y generar expectativas permitiendo que las organizaciones conduzcan su negocio de forma más práctica y ofrecer nuevos beneficios y servicios.

Un concepto erróneo que tienen inicialmente los emprendedores es que las aplicaciones son para grandes empresas, pero las apps son importantes en cualquier modelo de negocio sea para emprendedores o startup.

Desde el punto de vista metodológico, se asume un enfoque cualitativo que se combina con herramientas estadísticas para cuantificar la realidad estudiada. La investigación es de carácter descriptivo y causal, en el sentido que describe hechos que suceden en la empresa, se utiliza el método inductivo y deductivo, pues se inicia de premisas generales para llegar a conclusiones particulares. Como técnica se utiliza la entrevista apoyada en plataformas tecnológicas de videoconferencias, con los resultados obtenidos se procede al procesamiento y análisis de la información y conclusiones de las mismas.

Esto nos lleva como reto a indagar los beneficios del sistema operativo móvil del gigante google el cual es el dominante en el mercado, e investigar las herramientas de software, para después mediante la metodología de desarrollo de software más apropiada empezar con el diseño y desarrollo de la aplicación.

La palabra gestión, significa según la Real Academia Española, es hacer diligencias al logro de unos objetivos (Ramírez Cardona , 2010). A quien efectué diligencias para lograr un objetivo se le llama gestor. Gestor, es, pues, quien gestiona, es decir, quien ejecuta acciones para llegar a un resultado (Ramírez Cardona , 2010)

"Es la que involucra la coordinación y supervisión de las actividades de otros, de tal forma que esta se lleve a cabo de forma eficiente y eficaz" (Ramírez Cardona , 2010) La administración 
debe entenderse entonces como el conjunto de principios y técnicas que configuran una teoría, ciencia o arte que el hombre aplica en las actividades de planear, organizar, dirigir, coordinar y controlar (Ramírez Cardona , 2010)

La administración se define como el proceso de estructurar y utilizar conjuntos de recursos orientados hacia el logro de metas, para llevar a cabo las tareas en un entorno organizacional (Hitt, Black, \& Porter, 2006).

La ciencia se refiere al conjunto de conocimientos que se tiene sobre un fenómeno. En estricto sentido, ciencia es el conocimiento de las cosas por su causa (Ramírez Cardona , 2010).

La mayoría de los autores y tratadistas coinciden en afirmar que la administración es una ciencia nueva cuyo objeto y método de estudio aún se cuestionan pero, en todo caso, el fenómeno administrativo existe y debe seguirse observando con criterios y métodos científicos, como en el caso de las demás ciencias sociales (Ramírez Cardona , 2010)

Si la técnica es un conjunto de métodos y procedimientos, no podemos dudar que la administración es también una técnica, en la medida en que dichos método y procedimientos se aplican para la organización y ejecución de las actividades humanas administrativas (Ramírez Cardona , 2010).

Después, determinar las políticas, proyectos, programas, procedimientos, métodos, presupuestos, normas y estrategias necesarias para alcanzarlos, incluyendo además la toma de decisiones al tener que escoger entre diversos cursos de acción futura. En pocas palabras, es decir con anticipación lo que se quiere lograra en el futuro y el cómo se lo va a lograr (García del Junco, Martín Jimenez, \& Diez de Castro, 2001).

Es el hecho de influir en los individuos para que contribuyan a favor del cumplimiento de las metas organizacionales y grupales; por lo tanto, tiene que ver fundamentalmente con el aspecto interpersonal de la administración (Koontz \& Weihrich, 2004)

En cuanto al control consiste en medir y corregir el desempeño individual y organizacional para garantizar que los hechos se apeguen a los planes. Implica la medición del desempeño con base en metas y planes, la detección de desviaciones respecto a las normas y la contribución a la corrección de estas (Koontz \& Weihrich, 2004).

Se traduce la comercialización, como el acto de planear y organizar un conjunto de actividades necesarias que permitan poner, en el lugar indicado y en el momento preciso, una mercadería o servicio para que los clientes que conforman el mercado, lo conozcan y consuman (Díaz, 2014). 
La comercialización comprende las estrategias y los procesos para mover los productos desde el punto de fabricación hasta el punto de venta (Fleitman , 2000).

Los productos de consumo masivo se definen como productos de alta demanda. Son aquellos productos requeridos por los estratos de la sociedad, la cual cosa motiva a la competitividad entre empresas de este sector por la captación de público, intentando diferenciarse ofreciendo alternativas, precios o agregados (Kotler P. , 2006).

Es el acto de hacer que los productos estén disponibles para los clientes en las cantidades necesaria (Ferrel, Hirt, \& Ramos , 2004).

La eficacia se la define como "hacer las cosas correctas", es decir, las actividades de trabajo con las que la organización alcanza sus objetivos (Robbins \& Coulter, 2005).

La eficiencia consiste en obtener los mayores resultados con la mínima de inversión (Robbins \& Coulter, 2005).

Desarrollar una aplicación móvil para los clientes de una empresa ofrece grandes ventajas y oportunidades de negocio, y es un proyecto que requiere la intervención e implicación de un equipo de personas, la consideración de los factores desarrollados en este post y el apoyo y colaboración de profesionales del mundo digital. En un próximo artículo abordaré las aplicaciones móviles de empresa para uso interno y el uso de dispositivos móviles en la empresa. (Valentin, 2018)

Las aplicaciones móviles puedes servirte para ampliar tu mercado, como una nueva vía de comercializar tus productos y servicios, pero también puede servirte como complemento para tu nueva campaña de marketing. El desarrollo de aplicaciones móviles puede aplicarse como quieras a tu empresa, siempre y cuando tengas el objetivo claro. (Yeeply, 2016)

Gestionar un proceso administrativo bien definido se constituye como principio fundamental de la labor de un gerente, aunque dichas labores se realicen de forma empírica en la mayoría de las veces. Indistintamente, en las organizaciones, se debe trabajar por mejorar los procesos desarrollados en cada uno de los departamentos que la conforman, con el fin de orientar actividades hacia la optimización, un mejor desenvolvimiento del personal, mejora en tiempos de entrega de información interdepartamental, entre otros. (Falconi, 2019).

Estos programas de mobile e-commerce han probado su efectividad en empresas que ofrecen productos y servicios fuera del mundo digital. De esta forma, se diversifican los ingresos, se llega a un número más amplio de consumidores y se adquieren clientes asiduos. (Fernández, 2018). 
En la actualidad, los ordenadores personales son equipos indispensables y complementarios en labores cotidianas e industriales y desde su aparición, a finales de los años setenta, estos equipos informáticos han incorporado sistemas operativos para controlar su hardware. Sin embargo, en los últimos años, hemos visto un notable aumento en la proliferación de dispositivos móviles con capacidades muy similares a las de los ordenadores de escritorio y, además con software especializado para su contexto. (Serna, 2017)

Esta nos indica cuando una organización utiliza en forma productiva o económica sus recursos, esto nos indica que las organizaciones tiene que ser eficientes y eficaces, crear problemas y resolverlos, según la manera que sean administradas (Amaru, 2009).

En esencia, una aplicación no deja de ser un software. Para entender un poco mejor el concepto, podemos decir que las aplicaciones son para los móviles lo que los programas son para los ordenadores de escritorio. (Cuello, 2013).

Actualmente encontramos aplicaciones de todo tipo, forma y color, pero en los primeros teléfonos, estaban enfocadas en mejorar la productividad personal: se trataba de alarmas, calendarios, calculadoras y clientes de correo. (Cuello, 2013).

\section{MATERIALES Y MÉTODOS}

El presente artículo se estructura desde la concepción del mercado en línea, por tema de la pandemia mundial del covid-19, en especial por el requerimiento empresarial y sostenimiento en el mercado por el hecho de la pandemia, en el mismo se explican las principales acciones de mercado y para organizar las ventas y procesos internos de las necesidades del propio mercado interno y externo.

Mediante la sistematización conceptual de los términos que permiten comprender la existencia de energía en las acciones del mercado potencial y real, las mimas que procuran posicionar en la mente de los consumidores marcas, pero como toda energía, hay comportamientos y fuerzas que el mercado a pesar de ser el escenario en las que se desarrollan no pueden controlar, por lo cual nos referiremos al estudio de la realidad del entorno y su función social respecto a los clientes y la intención de permanencia de mercado.

Además, se aplicó un instrumento tipo encuesta al total de la muestra determinada, con el propósito de conocer en detalle y de primera mano la información respectiva. 
La población que será motivo de estudio del presente proyecto estará formada por el $48 \%$ de los compradores que utilizan su dispositivo móvil para buscar sus productos de interés que según indican las estadísticas de marketing móvil.

Entonces la población de estudio estará integrada por el $48 \%$ de la población del cantón Quevedo la cual será de 83.316 personas. Es la parte de la población que se selecciona para la investigación. Existiendo nuestro universo de investigación la muestra se determinará, tomando en consideración la población finita ya que esta es menor a 100.000. Los elementos de muestra serán 150 personas.

El tamaño de la muestra fue calculado con la siguiente ecuación:

$$
n=\frac{N \times Z_{a}{ }^{2} \times p \times q}{d^{2} \times(N-1)+Z_{a}{ }^{2} \times p \times q}
$$

Los elementos de la fórmula fueron:

$$
\begin{aligned}
& n=\text { Elementos de la muestra. } \\
& N=\text { Elementos del universo. } \\
& P / Q=\text { Probabilidad. } \\
& Z^{2}=\text { Nivel confianza }(90 \%=1,65 ; 95 \%=1,96 ; 99 \%=2,58) \\
& E=\text { Margen de error (director de estudio) } \\
& \text { Cuando valor de } P \text { y } Q \text { no se conozca, } P=Q=50(P=0,5 \text { y } Q=0,5) \\
& n=150 \\
& N=83.316 \\
& P / Q=0,5 . \\
& Z^{2}=1,96(95 \%) \\
& E=0,08
\end{aligned}
$$

\section{RESULTADOS}

Para la determinación de las acciones indicadas, permite a través del instrumento aplicado llegar a las siguientes aseveraciones. En la consulta de si la empresa cuenta la empresa con una cultura corporativa para el desarrollo de su actividad comercial, los resultados determinaron lo siguiente. 
Tabla1.

Cultura corporativa

\begin{tabular}{ccc}
\hline Categoría & Cantidad & Porcentaje \\
\hline Siempre & 34 & $23 \%$ \\
Casi siempre & 36 & $24 \%$ \\
A veces & 47 & $31 \%$ \\
Nunca & 33 & $22 \%$ \\
Total & $\mathbf{1 5 0}$ & $\mathbf{1 0 0 \%}$ \\
\hline
\end{tabular}

Fuente: Elaboración propia

Como se observa en la figura 1, para el 23 por ciento de los encuestados el conocimiento la Cultura Corporativa es poco adecuada, por cuanto al interior de las instituciones se da poca importancia a este tipo de aspecto, lo que redunda en escaso conocimiento del recurso humano respecto a las actividades empresariales, con un 24 por ciento casi siempre, esto es importante puesto que el 47 por ciento conoce de la existencia de la cultura Corporativa, mientras que para el 53 por ciento la conoce a veces o nunca ha sabido de esta situación, lo que implica que la empresa no involucra a los colaboradores en el esquema empresarial.

\section{Conocimmiento de la Cultura Corporativa Empresarial}

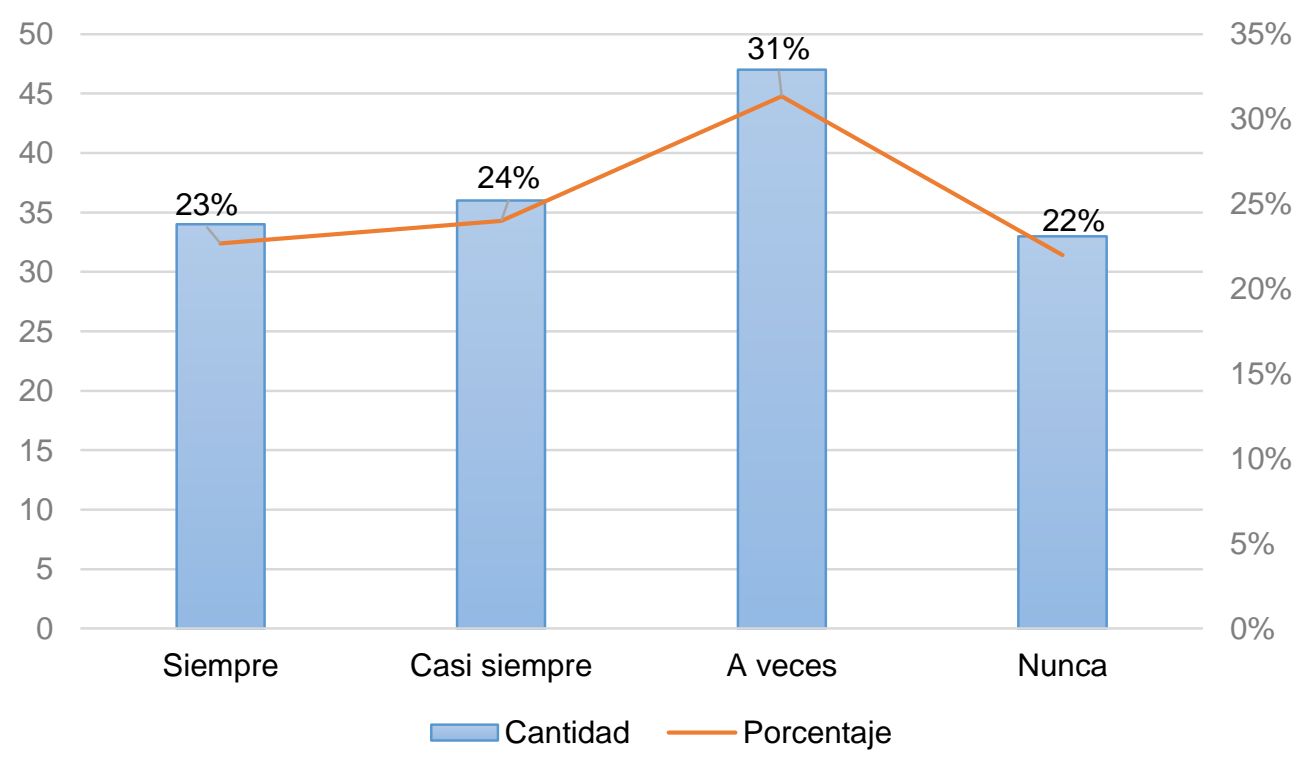

Figura 1. Conocimiento de la Cultura Corporativa Empresarial. Fuente: Elaboración propia

En cuanto a lo que la empresa considera de la definición de la visión en su planeación estratégica 
Tabla 2

Definición de la planeación estratégica empresarial.

\begin{tabular}{ccc}
\hline Categoría & Cantidad & Porcentaje \\
\hline Siempre & 20 & $13 \%$ \\
Casi siempre & 39 & $26 \%$ \\
A veces & 70 & $47 \%$ \\
Nunca & 22 & $15 \%$ \\
Total & $\mathbf{1 5 0}$ & $\mathbf{1 0 0 \%}$ \\
\hline
\end{tabular}

Fuente: Elaboración propia

Como se aprecia en la figura 2, para el 47 por ciento de los encuestados consideran que a veces apenas conocen de las empresas la Planeación Estratégica, siendo esto un alto porcentaje, que implica que las empresas no dejan conocer a sus colaboradores aspectos importantes para el desarrollo de la organización, seguido de un 15 por ciento de encuestado que manifestaron nunca conocer del aspecto, sumado a los encuestados de que conocen a veces esta situación la sumatoria de ambos representa el 62 por ciento de colaboradores con escaso conocimiento de la Planeación Estratégica, lo que no es conveniente por cuanto no se involucra a la actividad empresarial a los colaboradores de la empresa.

\section{Conocimiento de la Planeación Estratégica}

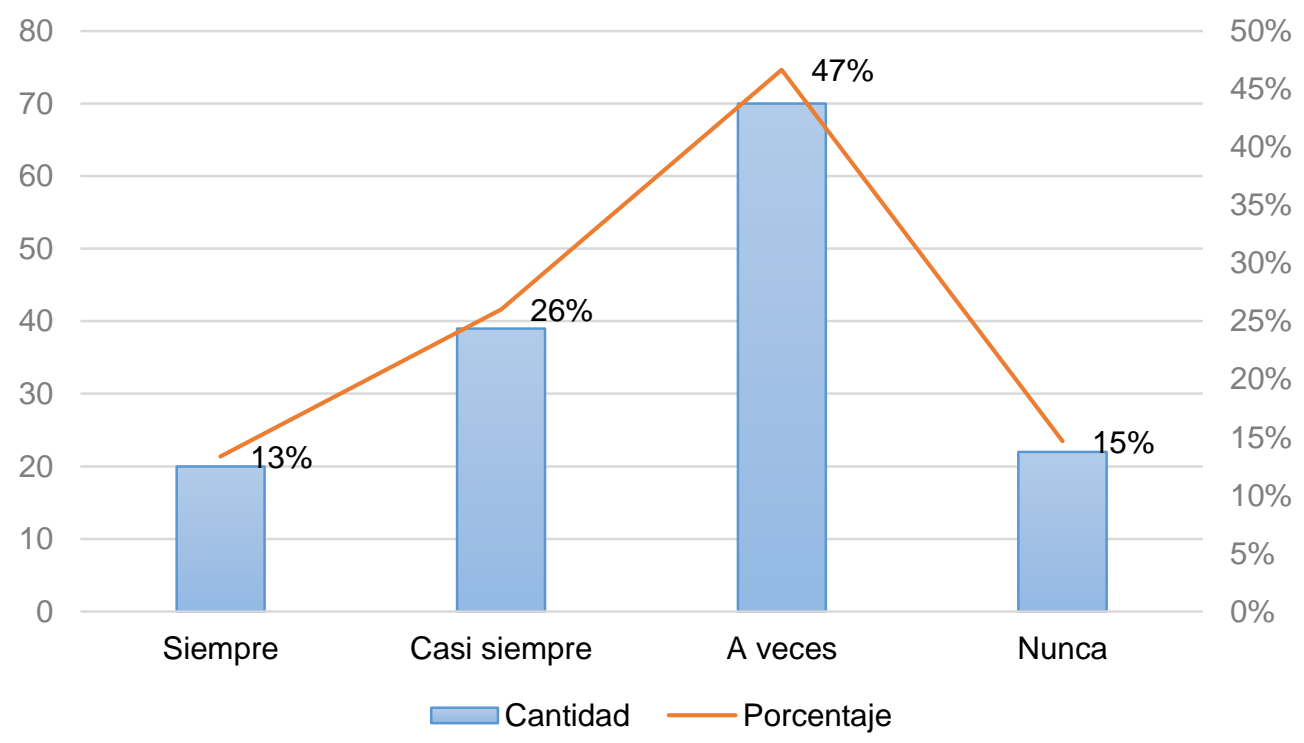

Figura 2. Conocimiento de la Planeación Estratégica. Fuente: Elaboración propia

Respecto a si cuenta la empresa con una clara definición de objetivos con enfoque innovador en su planeación estratégica, se obtuvo lo siguiente. 
Tabla 3

Conocimiento de los objetivos con enfoque innovador de la organización

\begin{tabular}{ccc}
\hline Categoría & Cantidad & Porcentaje \\
\hline Siempre & 9 & $6 \%$ \\
Casi siempre & 21 & $14 \%$ \\
A veces & 70 & $47 \%$ \\
Nunca & 50 & $33 \%$ \\
Total & $\mathbf{1 5 0}$ & $\mathbf{1 0 0 \%}$ \\
\hline
\end{tabular}

Fuente: Elaboración propia

Como se puede observar en la figura 3, respecto a lo que responde los encuestados se nota que para el 80 por ciento de estos desconocen el tema de los objetivos planteados por la organización, mientras que para el 6 por ciento manifiestan conocerlo y un 14 por ciento casi siempre lo conocen siendo un 20 por ciento de estos encuestados que conocen los objetivos del Plan Estratégico de la organización.

\section{Definición de Objetivos con enfoque innovador en la Planeación Estratégica}

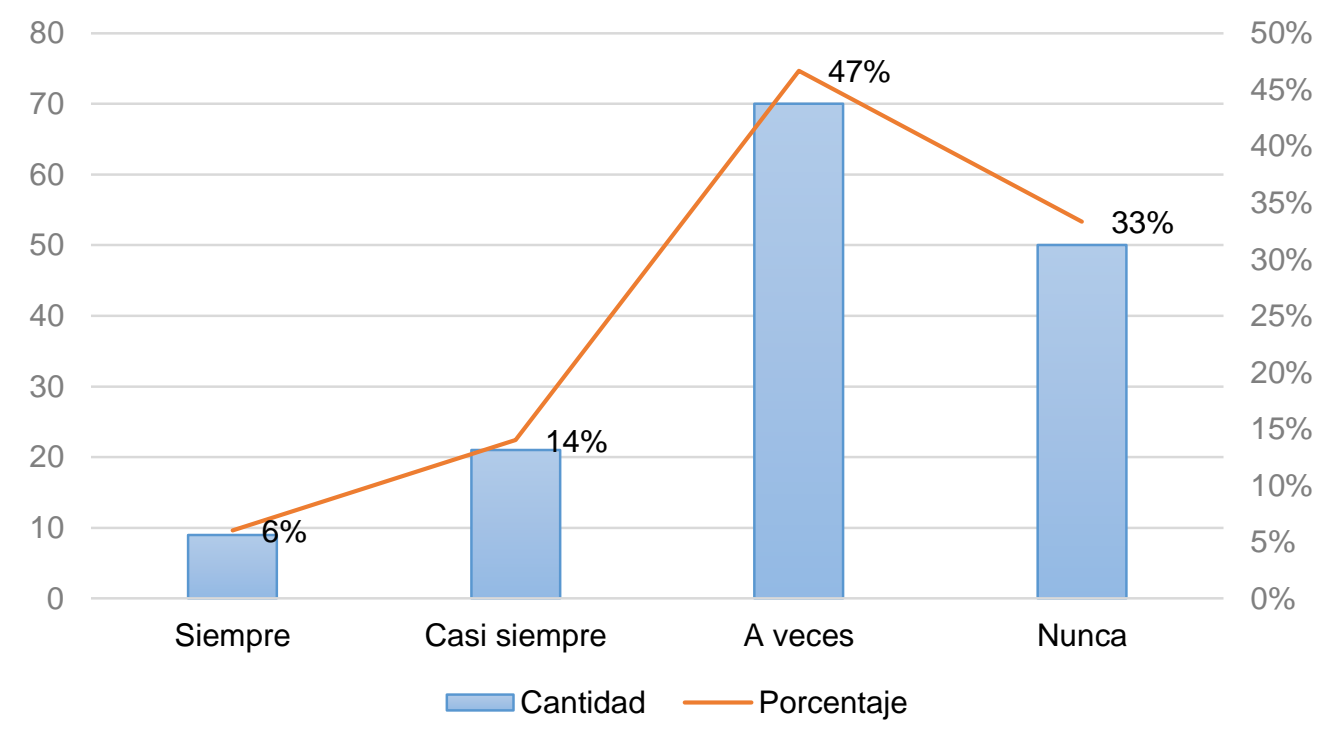

Figura 3. Definición de Objetivos con enfoque innovador para la planeación Estratégica. Fuente: Elaboración propia

Cuenta la empresa, definidas estrategias con enfoque innovador en su planeación estratégica 
Tabla 4.

Empresa con definición de estrategias con enfoque innovador

\begin{tabular}{ccc}
\hline Categoría & Cantidad & Porcentaje \\
\hline Siempre & 4 & $3 \%$ \\
Casi siempre & 20 & $13 \%$ \\
A veces & 78 & $52 \%$ \\
Nunca & 48 & $32 \%$ \\
Total & $\mathbf{1 5 0}$ & $\mathbf{1 0 0 \%}$ \\
\hline
\end{tabular}

Fuente: Elaboración propia

Como se observa en la figura 4. Para el 84 por ciento de los encuestados manifiestan conocer muy poco o nada de la estrategia son enfoque innovador, mientras que para apenas el 16 por ciento de estos considera que conocen de las estrategias, eso es negativo para la organización por cuanto existe una debilidad importante en cuanto al tema de estrategias.

\section{Estrategias con Enfoque Innovador}

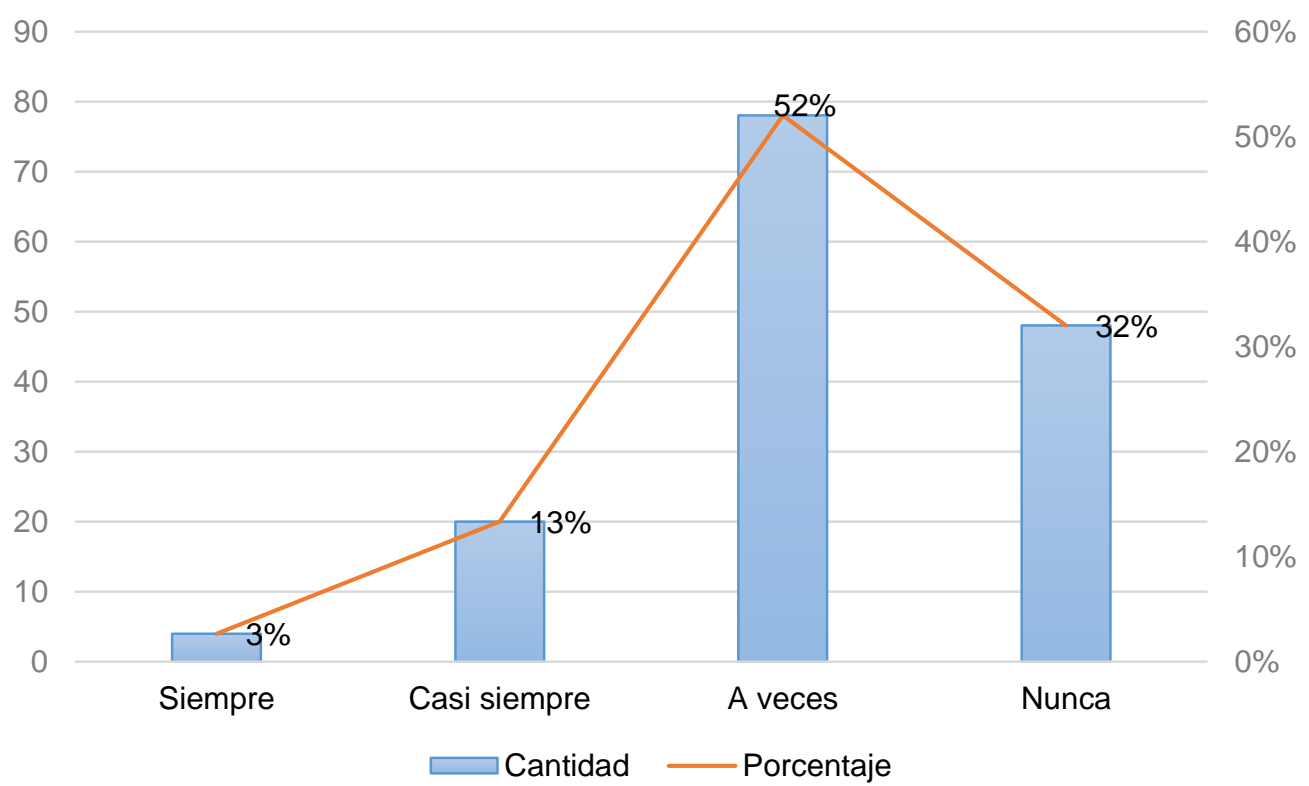

Figura 4. Definición de Estrategias con Enfoque Innovador. Fuente: Elaboración propia

Referente al criterio de los factores relevantes dentro del sistema de gestión administrativa de las empresas que utilizan aplicaciones móviles, se obtiene lo siguiente. 
Tabla 5

Sistemas relevantes de Aplicación Móvil para empresas

\begin{tabular}{ccc}
\hline Categoría & Cantidad & Porcentaje \\
\hline Sistemas de seguridad más confiables & 60 & $40 \%$ \\
Estructuración organizacional & 45 & $30 \%$ \\
Asistentes virtuales chatbots e inteligencia artificial & 45 & $30 \%$ \\
Total & $\mathbf{1 5 0}$ & $\mathbf{1 0 0 \%}$ \\
\hline
\end{tabular}

Fuente: Elaboración propia

En este apartado las personas encuestadas tuvieron una respuesta más paralela, donde el $40 \%$ de los encuestados dicen que el factor más relevante es el sistema de seguridad más confiable, ya que se debe tener todo con seguridad para que la gestión administrativa no sea alterada, sin embargo, el $30 \%$ de las personas encuestadas coinciden que se debe tener una estructuración organizacional adecuada para la gestión administrativa de las empresas con aplicaciones móviles. Del mismo modo, el otro 30\% de las personas encuestadas aseguran que el factor más importante son los asistentes virtuales chatbots e inteligencia artificial para las empresas con aplicaciones móviles

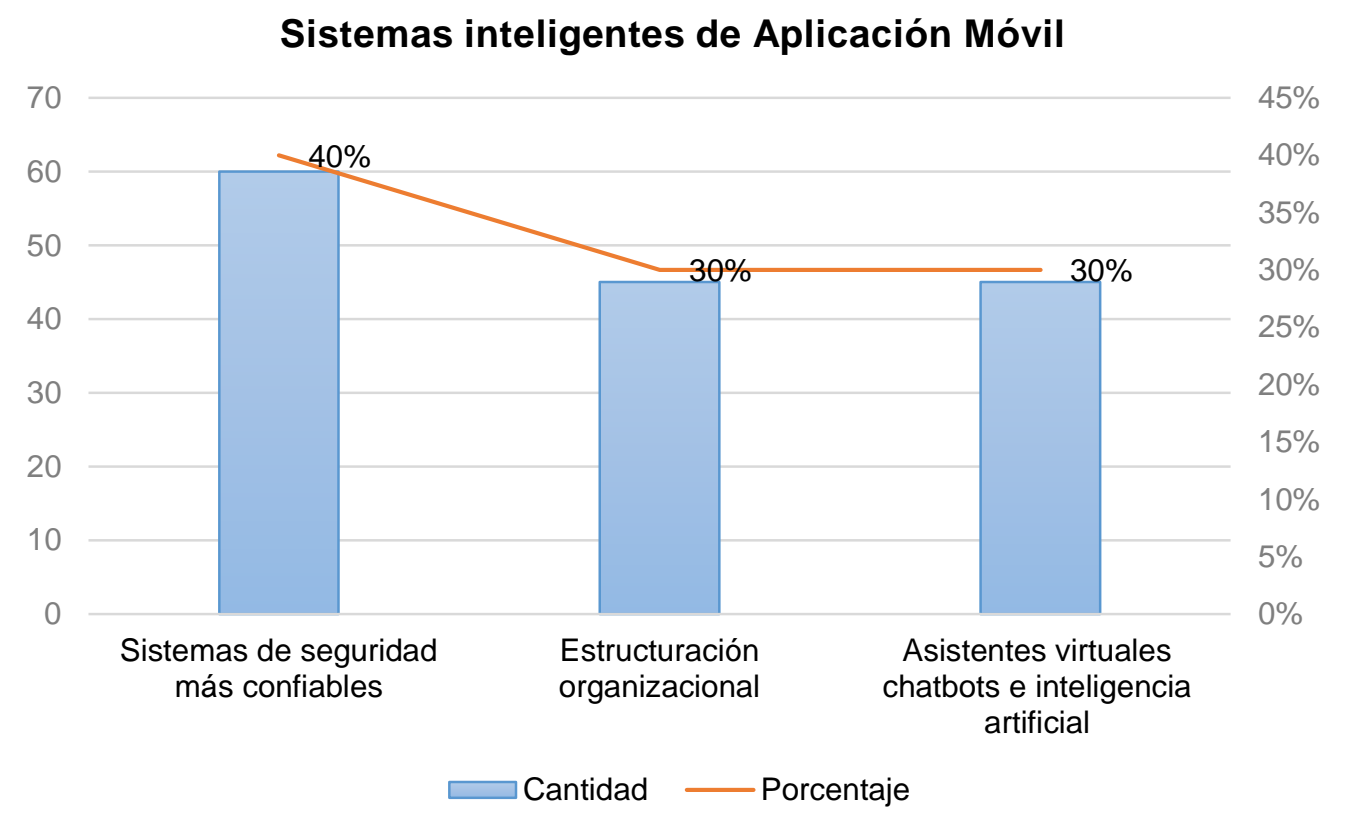

Figura 5. Aplicación de sistemas de Gestión Administrativas. Fuente: Elaboración propia

Respecto al sistema de gestión administrativa es importante el control micro organizaciones de las empresas de usos móviles. Lo que se refiere a los indicadores que forman parte del control. 
Tabla 6

Indicadores de control del sistema de gestión administrativa

\begin{tabular}{ccc}
\hline Categoría & Cantidad & Porcentaje \\
\hline Ventas perdidas & 24 & $\mathbf{1 6 \%}$ \\
Reclamos por despacho perdido & 66 & $44 \%$ \\
Desempeño laboral & 46 & $31 \%$ \\
Rotación del personal & 14 & $9 \%$ \\
Total & $\mathbf{1 5 0}$ & $\mathbf{1 0 0 \%}$ \\
\hline
\end{tabular}

Fuente: Elaboración propia

Dado los datos de la figura 6, muestra que 24 personas es decir el 16\% que unos de los indicadores para verificar un adecuado control en el sistema de gestión administrativa son las ventas perdidas, deduciendo por lo tanto que es necesario que cada empresa tenga un monitoreo constante de las ventas, mientras que 66 personas es del $44 \%$ respondiendo que es necesario tener un control del reclamo de despacho perdido, en cambio el $36 \%$ es decir 46 personas manifestaron que el desempeño del personal tiene que ser un indicador de control administrativo para poder estar al tanto de sus ventas y rentabilidad, y por ultimo 14 personas es decir el 9.30\% que la rotación del personas es decir el despido o cambio puedo influir en el control del sistema de gestión administrativo.

\section{Indicadores de Gestión}

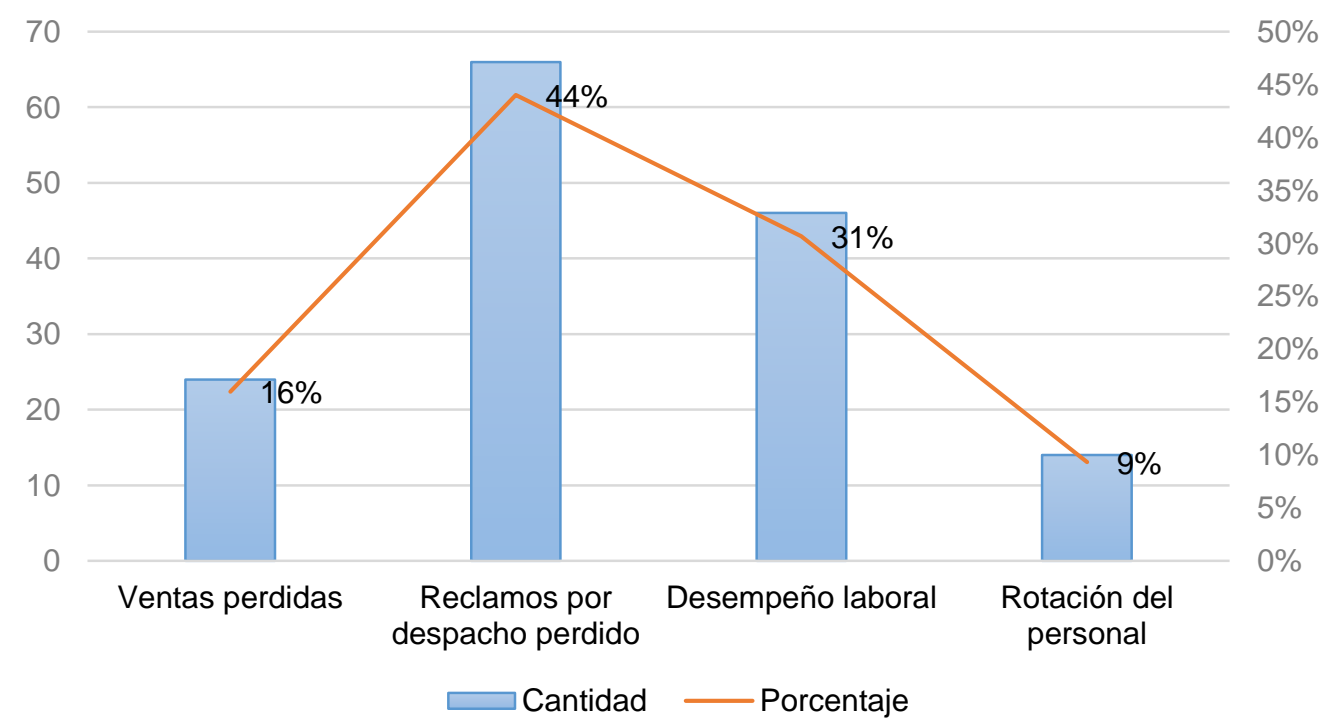

Figura 6. Indicadores de Gestión Administrativa. Fuente: Elaboración propia 


\section{CONCLUSIONES}

El rendimiento alcanzado por las empresas que comercializan productos de consumo masivo mediante aplicaciones móviles durante el periodo 2019 - 2020 ha tenido muchos efectos positivos sobre las empresas, todo esto causado por la dificultad respecto a la pandemia, que se presentó durante el periodo al momento de realizar comprar en modalidad presencial, esta forma de comercialización fue una excelente salida para poder abastecer los mercados manteniendo la bio-seguridad.

Aunque esta modalidad de comercialización haya tenido tantos efectos positivos para la empresa, no ha incrementado de tal manera los puestos de trabajo, aunque fueron requeridas más personas para mover el volumen de productos, no es en la proporción que han crecido las ventas, por lo que las empresas deberían buscar áreas en las cuales contratar para poder mantener este volumen de comercialización luego de la situación de confinamiento.

Un sistema de Gestión Administrativas que usan aplicaciones móviles, es esencial conocer el grado de eficiencia de las aplicaciones móviles, se han posicionado como una de las herramientas más eficaces de la empresa. Gracias a la tecnología los servicios que se ofrece pueden ser más eficientes a la hora de salir al mercado, la innovación existente en un mundo globalizado permite que cada vez se mejore la calidad de los sistemas que utilizan la mayoría de las empresas, esto con el propósito de que los clientes tengan una mayor acogida y una sensación de conformidad.

Las aplicaciones han permitido un cambio en la dinámica del mercado es por ella, que la implementación de estos sistemas como parte del modelo del negocio es uno de los pilares fundamentales de crecimiento y desarrollo. Es indispensable apostar por nuevas herramientas de trabajo que permitan llevar a un giro el negocio, sin lugar a dudas la pandemia del Covid 19 fue una de las razones por las que también las apps móviles cobraron una mayor participación, si bien es cierto aun no todas las personas tienen acceso a este tipo de aplicaciones, sin embargo, si existe una alta cantidad de personas que usan esta herramienta, unos más que otros, pero se debe por el ambiente en el que se desarrollan.

\section{REFERENCIAS BIBLIOGRÁFICAS}

Amaru, A. (2009). Fundamentos de administracion, Teoria general y procesos administrativos.

Andersen, A. (2002). Mercadeo Social, Desarrollo Social y Medio Ambiente. Journal of Public Policy \& Marketing, 21(1). 
Ayllón, L. (2012). Marketing Ecológico y Social. Universidad Nacional Del Centro Nacional del Perú.

Boza, J., Mendoza, E., Manjarrez, N., \& Escobar, H. (2018). Innovación tecnológica y competitividad empresarial con responsabilidad social: Factores estratégicos en los emprendimientos de la Provincia de Los Ríos, Ecuador. MEMORALIA, Especial (Congreso CEAC 2018 ESPE Latacunga).

Cuello, J. (2013). Diseñando Apps para móviles. Argentina: Edición Catalina Duque Giraldo.

Díaz, J. A. (2014). Comercialización de los productos y servicios de la ciencia: retos y perspectiva. Revista Cubana de Ciencia Agrícola.

Falconi, J. (2019). Gestión administrativa: Estudio desde la administración de los procesos de una empresa. Cuenca: Visionario Digital.

Fernández, H. (2018). La importancia de las apps móviles en las empresas. Obtenido de La importancia de las apps móviles en las empresas: https://economiatic.com/importanciaapps-moviles-empresas/

Ferrel, Hirt, G., \& Ramos, L. (2004). Introducción a los Negocios en un Mundo Cambiante. Mc Graw Hill.

Fleitman, J. (2000). Negocios Exitosos. Mc Graw Hill.

García del Junco, J., Martín Jimenez, F., \& Diez de Castro, E. (2001). Administración y Dirección. McGraw-Hill Interamericana.

Hitt, M., Black, S., \& Porter, L. (2006). Administración. Pearson.

Koontz, H., \& Weihrich, H. (2004). Administración Una Perspetiva Global. McGraw-Hill Interamericana.

Kotler, P. (2001). Dirección de marketing (10ma. ed.). México: Pearson Prentice Hall.

Kotler, P. (2006). Marketing. Mexico: Person.

Kotler, P., \& Armstrong, G. (2003). Fundamentos de marketing (6ta. ed.). México: Pearson Educación.

Pérez, L. A. (2004). Marketing Social: teoría y práctica. Pearson Educación. doi:9702605415, 9789702605416

Ramírez Cardona, C. (2010). Fundamentos de Administración. Bogotá. 
Robbins, S., \& Coulter, M. (2005). Administracion. Pearson.

Serna, S. (2017). Diseño de Interfases en Aplicaciones Moviles. Bogota: Rama.

Valentin. (2018). Estrategia móvil para las empresas. Obtenido de Estrategia móvil para las empresas: https://www.ttandem.com/blog/empresas-aplicaciones-moviles/

Yeeply. (08 de 02 de 2016). Desarrollo de apps móviles para empresas. Obtenido de https://www.yeeply.com/blog/desarrollo-de-aplicaciones-moviles-empresas/ 